\title{
EL REGISTRO GEOLÓGICO INÉDITO DE BONPLAND SOBRE LA TURBA MALVINERA
}

\author{
Bonpland's unprecedented geological record of the malvinera peat
}

\author{
DAVID N. F. GUEVARA' ${ }^{1}$
}

\begin{abstract}
Resumen: El presente trabajo se centró en la identificación y puesta en valor histórico de los registros geológicos, vinculados a las Islas Malvinas y Tierra del Fuego, Argentina, que enumera y describe en su Catálogo Geológico, individualizado como manuscrito MS 210, el naturalista francés Aimé Bonpland (1773-1858). Éstos fueron examinados por primera vez por esta investigación en el año 2018 durante un estudio sobre los Archivos Bonpland no digitalizados y depositados en el Museo Nacional de Historia Natural, París, Francia. Los registros mencionados tuvieron su origen en las muestras geológicas entregadas por el Sr. Luis Vernet (1791-1871), primer gobernador y comandante argentino de las Islas Malvinas, cuando ambos coincidieron con su presencia en Buenos Aires en el año 1832. Estas muestras fueron estudiadas, clasificadas e incorporadas al Catálogo de Geología General del Museo Nacional de Historia Natural de París, como turba de color marrón de las Islas Malvinas, y rocas de la Isla de los Estados, pertenecientes a la Colección Bonpland y como país de origen Argentina. Del mismo modo, se documenta el mencionado catálogo geológico y se analizan las cartas de Bonpland a Humboldt de 1832, en orden al contexto e interés que las mismas representan para la historia de la República Argentina.
\end{abstract}

Palabras clave: Argentina, Catálogo Geológico, Humboldt, Isla de los Estados, Islas Malvinas, Tierra del Fuego.

Summary: The present work focused on the identification of and the historical value given to the geological records, linked to the Malvinas Islands and Tierra del Fuego, Argentina, which the French naturalist Aimé Bonpland (1773-1858) enumerates and describes in his geological catalogue, individualized as manuscript MS 210. These were examined for the first time by this investigation in the year 2018 during a study of Bonpland's non digitalized Files at the National Museum of Natural History, Paris, France. The aforementioned records had their origin in the geological samples given by Mr. Luis Vernet (1791-1871), the first governor and Argentinian commandant of the Malvinas Islands, when both of them were in Buenos Aires in the year 1832. Samples were classified and incorporated in the General Geology Catalog of the National Museum of Natural History in Paris as brown peat from the Malvinas Islands and rocks from Isla de los Estados, belonging to the Bonpland Collection with Argentina as a country of origin. At the same time, the aforementioned geological catalogue is documented, and Bonpland's 1832 letters to Humboldt are analyzed, in relation to the context and interest that those letters represent for the history of the Argentine Republic.

Key words: Argentina, Geological Catalogue, Humboldt, Malvinas Islands, Isla de los Estados, Tierra del Fuego.

\footnotetext{
${ }^{1}$ Historiador de Tierra del Fuego e Investigador Numismático. Pioneros Fueguinos 351 PB "A" (CP9420) Río Grande, Provincia de Tierra del Fuego, Antártida e Islas del Atlántico Sur, República Argentina. E-mail: davidtdf@hotmail. com / elalquimistatdf@gmail
} 


\section{Introducción}

En este trabajo se menciona por primera vez el hallazgo de registros geológicos inéditos, vinculados a las Islas Malvinas y Tierra del Fuego, Argentina, en el Catálogo Geológico del naturalista francés Aimé Bonpland (17731858) (Fig. 1A), o Don Amado (Cerruti, 2013), como también se lo conoce en la Provincia de Corrientes, Argentina. Este manuscrito conservado como MS 210, en la Biblioteca Central del Museo Nacional de Historia Natural de París, Francia, que el autor de este trabajo, documentó in situ durante abril y mayo del 2018 (Fig. 1B). Labor realizada en el marco de una investigación, sobre los archivos de Bonpland no digitalizados, siguiendo los escritos, entre otros, de la prestigiosa botánica Alicia Lourteig, sobre los manuscritos de Bonpland conservados en el Museo Nacional de Historia Natural de París (Lourteig, 1977).

Los mencionados registros geológicos, se corresponden con muestras entregadas a Bonpland por el Sr. Luis Vernet (1791-1871), primer gobernador y comandante argentino de las Islas Malvinas, "Donné par Mr. Vernet qui a éte Comandant des Isles Malonuines en 1831" (Bonpland, 1858). Oportunidad en que ambos coincidieron con su presencia en Buenos Aires en 1832; y en momentos que Bonpland preparaba sus colecciones para el envío al Museo Nacional de Historia Natural y su entrega al Sr. Mandeville, entonces cónsul francés en Buenos Aires, de 25 cajas que contenían plantas secas, rocas, cortezas y raíces medicinales, pájaros y otros objetos relacionados con la historia natural del Paraguay, Brasil, Uruguay y Argentina (Hamy, 1906).

Bonpland en 1832, durante su permanencia en Buenos Aires, escribió cinco cartas a su amigo y compañero de viaje a las tierras equinocciales (1799-1804), el naturalista y geólogo alemán Alexander von Humboldt (1769-1859), donde se mostró preocupado, acerca de los acontecimientos que ocuparon al Gobierno Argentino y al de los Estados Unidos, por los incidentes ocurridos en 1831 en las Islas Malvinas (Hossard, 2016). Circunstancias que
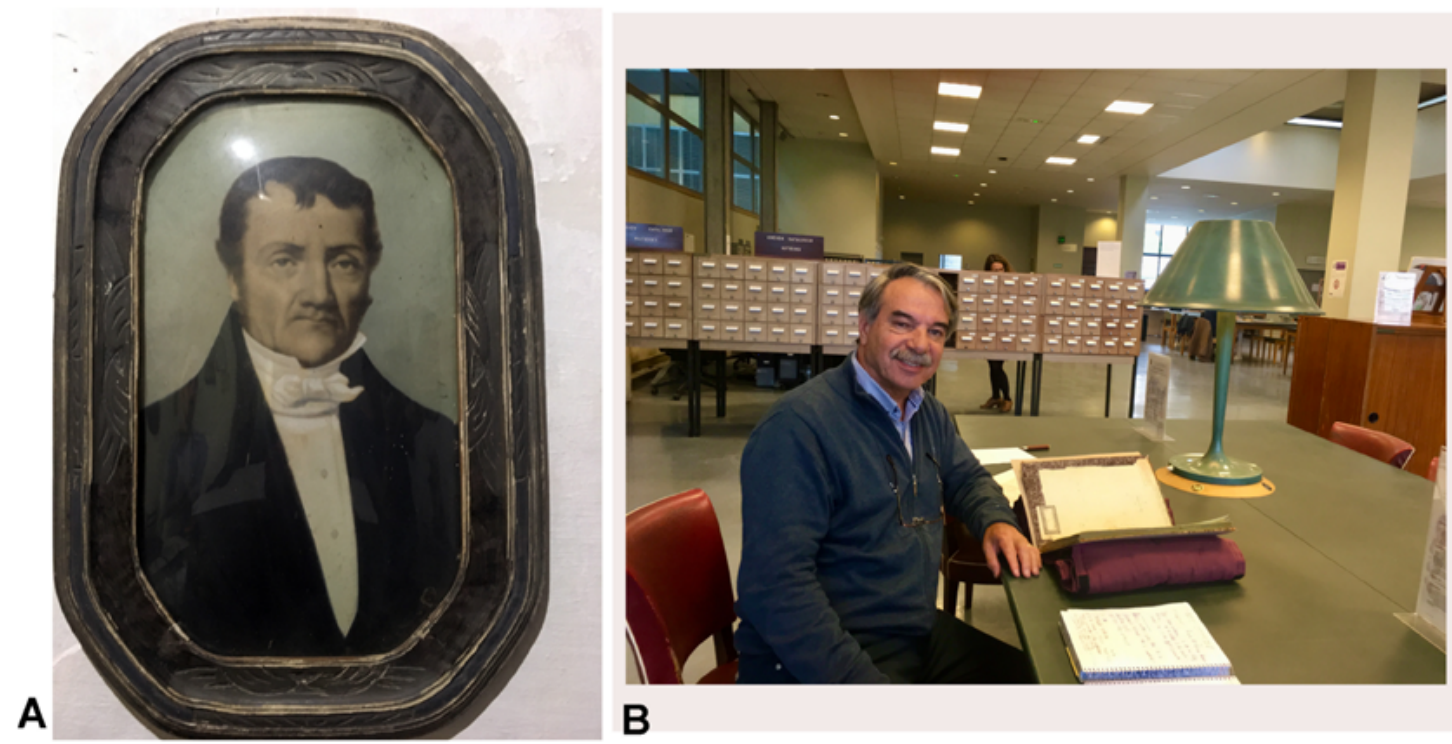

B

Fig. 1. A: Aimé Bonpland (1773-1858), Cuadro, Estancia El Recreo, Santa Ana del Uruguay, actual localidad de Bonpland, Departamento de Paso de los Libres, Corrientes, Argentina. B: David Nelson Federico Guevara en la Biblioteca Central del Museo Nacional de Historia Natural, París, Francia, 2018.

Fig. 1. Aimé Bonpland (1773-1858), Painting, El Recreo Ranch, Santa Ana del Uruguay, current city of Bonpland, Paso de los Libres, Corrientes, Argentina. B: David Nelson Federico Guevara Central Library of the National Museum of Natural History, Paris, France, 2018. 
fueron relevantes para el estudio de la historia de la Provincia de Tierra del Fuego, Antártida e Islas del Atlántico Sur, Argentina (Fig. 2). El antecedente más cercano que se conoce en relación al sabio francés en suelo fueguino, es la existencia de un río que lleva su nombre, Río Bonpland (Guevara, 2016), y una calle del ejido urbano de la ciudad de Río Grande, Tierra del Fuego, Argentina.

El objetivo de esta contribución es dar a conocer el hallazgo, identificación y puesta en valor histórico de estos registros geológicos, vinculados a las Islas Malvinas y Tierra del Fuego, Argentina, en el Catálogo Geólogico de Aimé Bonpland, y comprobar si los mismos fueron clasificados en el Catálogo General de Geología del museo antes mencionado. También describir el mencionado catálogo geológico, y metodología aplicada en la toma

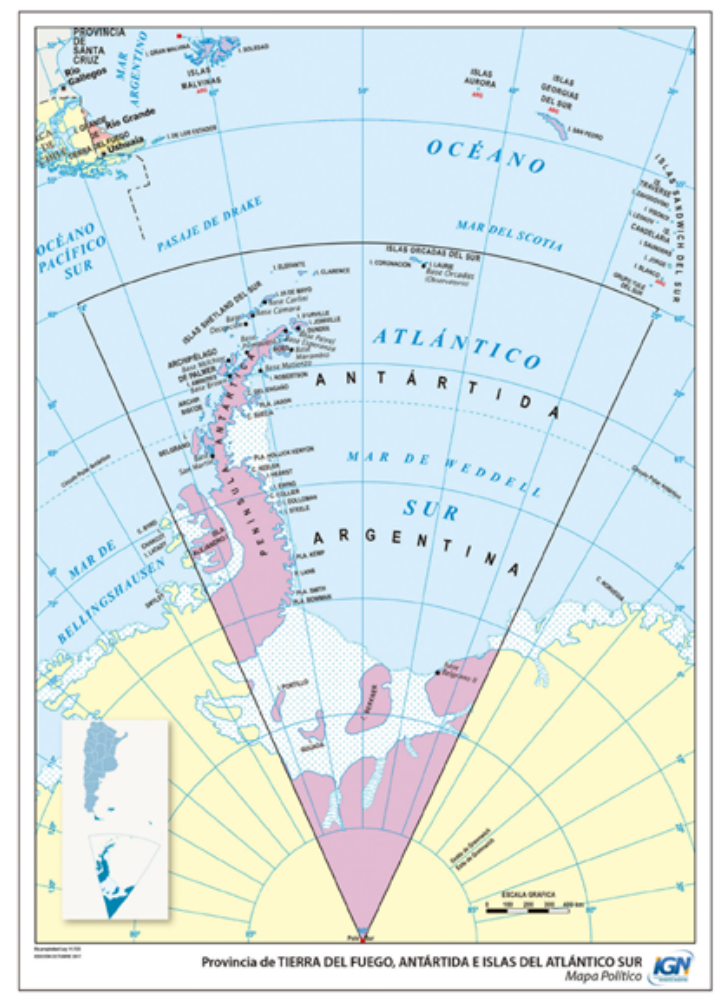

Fig. 2. Provincia de Tierra del Fuego, Antártida e Islas del Atlántico Sur, Argentina. Instituto Geográfico Nacional, 2020. Fig. 2. Tierra del Fuego Province, Antarctica and South Atlantic Islands, Argentina. National Geographic Institute, 2020 . de muestras para su registración en el mismo. Finalmente, en relación al intercambio epistolar de Bonpland a Humboldt, dar a conocer sus preocupaciones en orden al catálogo y si tuvo intenciones de viajar a las costas de Patagonia y a las Islas Malvinas.

\section{Materiales y Métodos}

El autor de esta investigación, a partir del día 27 de abril de 2018, acompañado del traductor e intérprete franco-argentino, Robinson Savary, se presentaron en la Dirección de Bibliotecas y Documentación, de la Dirección General de Colecciones de la Biblioteca Central del Museo Nacional de Historia Natural de París. En ese lugar cumplimentó la documental personal y profesional, correspondiente a las autorizaciones previamente tramitadas, y la acreditación en Sala de Reserva, donde se consultan las colecciones patrimoniales (archivos, manuscritos, libros raros, documentos iconográficos), en el espacio reservado a la sala de investigación del segundo piso, Sala 2 de Investigaciones. Se accedió finalmente a los manuscritos de Aimé Bonpland, dispuestos para su estudio, bajo la supervisión de la curadora, Sra. Véronique Van de Ponseele.

El corpus que constituye la fuente primaria seleccionada para esta investigación, es el Catálogo Geológico de Aimé Bonpland, que lleva como título: "Catalogue pour servir a la Géologie des cótes de l'Uruguay, du Parana, de la plata; de toutes les missions jesuitiques, des provinces du Paraguay, de Corrientes, de l'Entre-Rios de la Cisplatine et de la république argentine, par Aimé Bonpland" (Fig. 3A). El mismo está integrado por 357 registros que guardan cronología con sus viajes, y la estructura es similar a su Diario de Botánica, "Journal de Botanique", en 84 páginas, numeradas de puño letra del sabio, donde enumera y describe cada muestra geológica, sean rocas, minerales o fósiles.

El abordaje de la obra de Bonpland, a través de este histórico manuscrito, individualizado como MS 210, refleja el largo camino geológico transitado por el naturalista en sus viajes por la América Meridional, cuya conservación inicial 

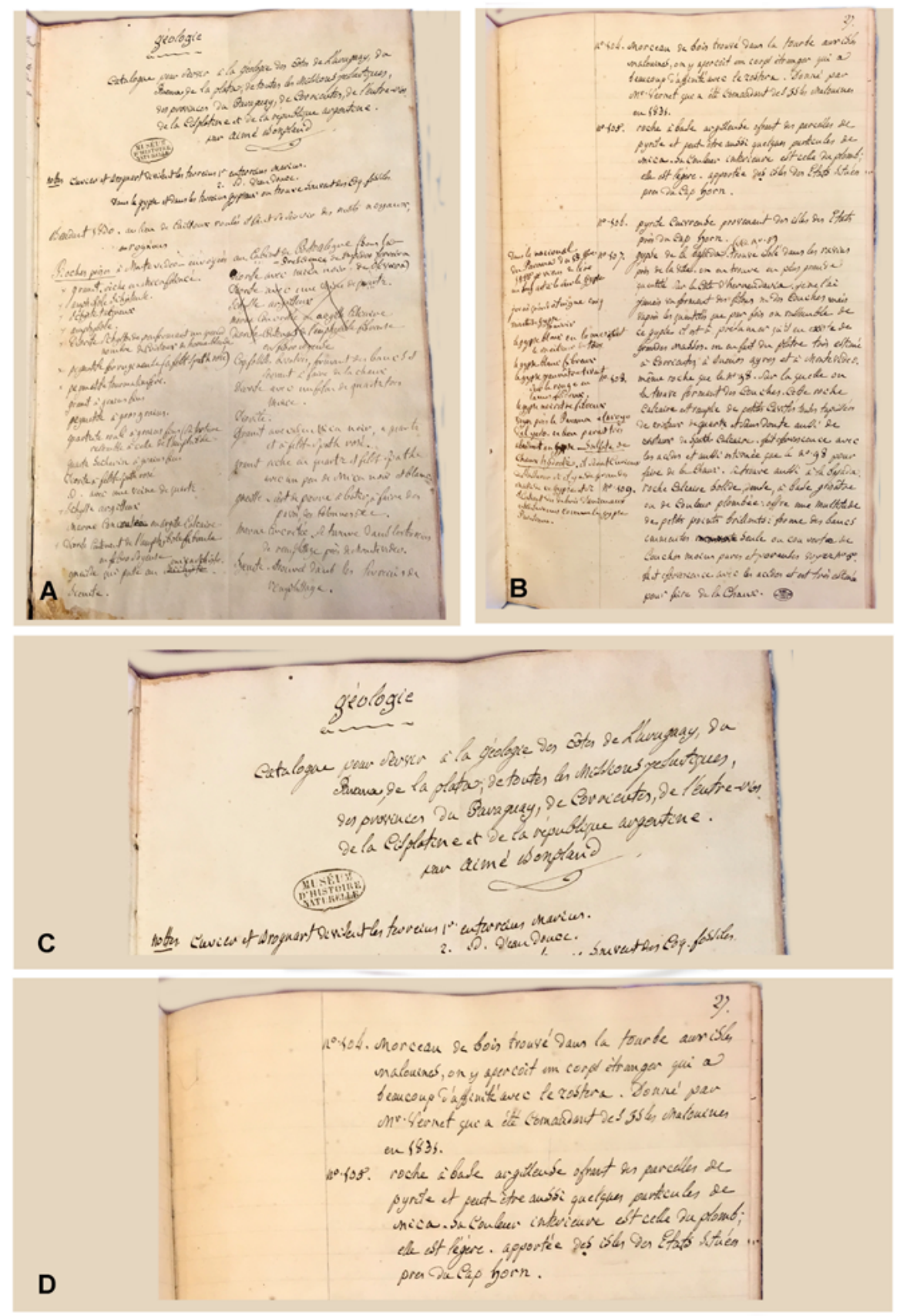

Fig. 3. Catálogo Geológico, Bonpland, 1858. MS 210, Biblioteca Central del Museo Nacional de Historia Natural, París, 2018. A: Geología, primera página, completa, sin número. Lista de rocas. B: Página 27, completa, $\mathrm{N}^{\circ} 104$ Islas Malvinas, $\mathrm{N}^{\circ} 105$ Isla de los Estados, $\mathrm{N}^{\mathrm{o}} 106$ Isla de los Estados, $\mathrm{N}^{\mathrm{o}} 107, \mathrm{~N}^{\circ} 108$, importante nota marginal. C: Título, Geología, descripción catálogo geológico, firma Bonpland. D: Página 27, No 104 Islas Malvinas, Vernet, 1831, № 105 Isla de los Estados. Tierra del Fuego, Argentina.

Fig. 3. Geological Catalog, Bonpland, 1858. MS 210, Central Library of the National Museum of Natural History, Paris, 2018. A: Geology, first page, complete, unnumbered. List of rocks. B: Page 27, complete, Number 104 Malvinas Islands, Number 105 Isla de los Estados, Number 106 Isla de los Estados, Number 107, Number 108, important marginal note. C: Title, Geology, description of the geological catalog, signature Bonpland. D: Page 27, Number 104 Malvinas Islands, Vernet, 1831, Number 105 Isla de los Estados, Tierra del Fuego, Argentina. 
estuvo al cuidado del geólogo Jules Desnoyers (1800-1887), bibliotecario del museo de París, junto a otros documentos inéditos del sabio (Brunel, 1871), que fueron entregados en 1858 por la familia Perichon de Corrientes, Argentina, al Conde de Brossard, Cónsul de Francia en Asunción del Paraguay (Hamy, 1906).

Bonpland, luego de su temprano antecedente minero en busca de minas de mercurio durante su confinación en el Paraguay (1821-1831), se volcó a partir de 1832, a la prospección de rocas, minerales y fósiles (Guevara, 2019), sin otra formación, en materia de estudios geológicos, que la aprendida en su estrecha relación durante el viaje a las tierras equinocciales con Alexander von Humboldt. Este último estaba formado como geólogo en la Academia de Minería de Freiberg, Alemania, bajo la dirección de Abraham Gottlob Werner (17491817), autor de un Manual de Orictognosia para quien interesa la mineralogía, en 1792, aunque no se conoce del naturalista alemán un diario específico de mineralogía ${ }^{2}$.

Por lo antes expuesto, es probable que los métodos de Bonpland, pudieron tener origen en esa experiencia, aplicados para la recolección de muestras, que consistió en recoger seis muestras de cada roca que tomó del tamaño de un puño, tal cual lo expresó en su carta a Humboldt, fechada en Corrientes, el 28 de marzo de 1838 (Hamy, 1906) “j'ai toujours eu soin de collecter six échantillons de chaque roche; je les ai pris de la grosseur du poing, ils sont bien choisis et bien emballés". Incluso en la misma correspondencia, entre otras cosas, lamentó que todavía no haya visto su catálogo de rocas que le envió; ya que el mismo constituye la parte geológica de este sector de América que visitó a partir de 1817.

En este orden de cosas, se procedió a relevar y documentar minuciosamente, el Catálogo Geológico de Bonpland 1858, en particular los registros que resultaron de

2 Comunicación personal, mayo de 2020, con el Dr. Alberto Gómez Gutiérrez, autor de Humboldtiana neogranadina, 2018, que recopila el trabajo y observación del viajero durante su estadía en la Nueva Granada, y que reúne en cuatro tomos comentarios, ilustraciones y estudios inéditos de y acerca de la obra de Humboldt. mayor interés, conforme a los antecedentes históricos, geográficos, cartas y bibliografía, seleccionada al efecto. Del mismo modo, se incorporó a la tarea, el valor agregado dado por la experiencia previa de esta investigación, en la indagación histórica en los propios espacios geográficos que transitó Bonpland, por ejemplo, Itá Pucú en 1834, y La Cruz-Tres Cerros entre 1854 a 1856, en la Provincia de Corrientes, Argentina; finalmente, todo ello permitió obtener los resultados que se presentan a continuación.

\section{Resultados y Discusión}

En la página 27 (Fig. 3B), de este Catálogo Geológico de Bonpland (Fig. 3C), se constató que el registro $\mathrm{N}^{\mathrm{o}} 104$, refiere a una muestra de las Islas Malvinas (Fig. 3D); y los registros subsiguientes, $\mathrm{N}^{\mathrm{o}} 105$ y $\mathrm{N}^{\circ} 106$, tratan sobre rocas provenientes de la Isla de los Estados, Tierra del Fuego, Argentina. Del mismo modo, en la página 36 (Fig. 4A), luego del registro $\mathrm{N}^{\mathrm{o}} 154$, anota que terminó el catálogo en Buenos Aires, el 26 de diciembre de 1836, y que envía dos copias a Europa, por una parte toda la colección y el catálogo al museo de París, y el catálogo solamente a Humboldt, todo con la firma autógrafa de Bonpland, fechada 26 de diciembre de 1836. Acto seguido continúa en la misma página, y escribe una leyenda que dice que todos los objetos que siguen han sido incorporados después del 26 de diciembre de 1836 (Fig. 4B), y a continuación el registro $\mathrm{N}^{\mathrm{o}} 155$, hasta el último de los registros en 1857, que corresponde al $\mathrm{N}^{\mathrm{o}} 357$, página 84 .

En relación al registro $\mathrm{N}^{\circ} 104$, se señala que describe la muestra dada por el Sr. Vernet, que ha sido Gobernador de las Islas Malvinas en 1831, como un trozo de madera encontrado en la turba de las Islas Malvinas, donde hay un cuerpo extraño que tiene afinidad con la Zostera marina L.; cuya transcripción literal del registro original dice: " $\mathrm{N}^{\mathrm{0}} 104$. Morceau de bois trouvé dans la tourbe aux Iles Malouines, on y apercoit um corpos ètranger qui a beaucoup d'affinité avec le zostera. Donné par Mr. Vernet qui a éte Comandant des Isles Malonuines en 1831". 

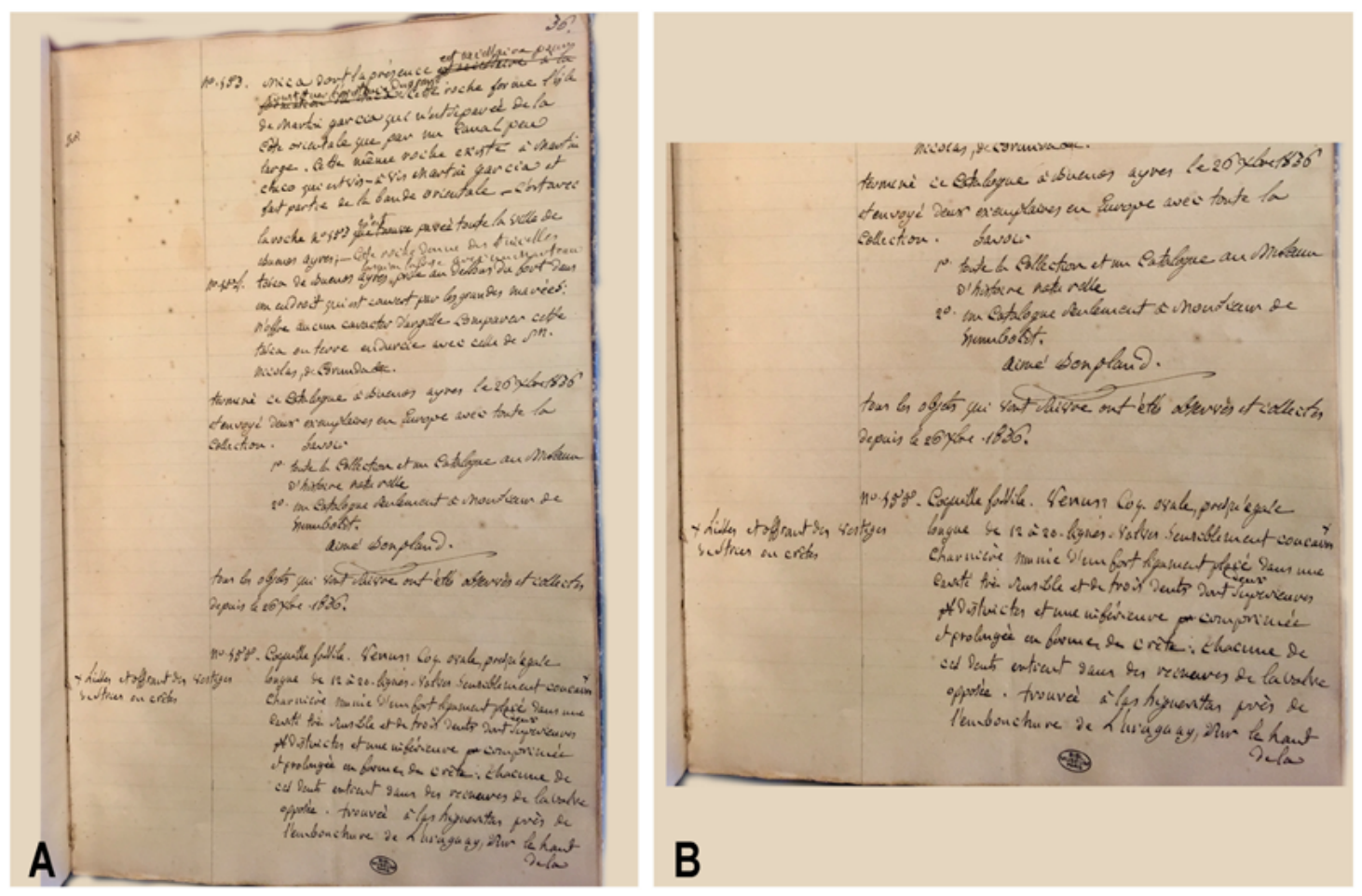

Fig. 4. Catálogo Geológico Bonpland, 1858. MS 210, Biblioteca Central del Museo Nacional de Historia Natural, París, 2018. A: Página 36, completa, $\mathrm{N}^{\mathrm{o}} 153, \mathrm{~N}^{\mathrm{o}} 154$, notas, Buenos Aires, 1836, firma Bonpland, $\mathrm{N}^{\mathrm{o}} 155$. B: $\mathrm{N}^{\mathrm{o}} 154$, nota de envío de dos ejemplares del Catálogo Geológico a Europa, $1^{\circ}$. al Museo de Historia Natural de París, $2^{\circ}$. a Humboldt, Buenos Aires, 26 de diciembre de 1836, firma Bonpland. Nota de continuación del catálogo, $\mathrm{N}^{\mathrm{o}} 155$.

Fig. 4. Bonpland Geological Catalogue, 1858. MS 210, Central Library of the National Museum of Natural History, Paris, 2018. A: page 36, complete, Number 153, Number 154, notes, Buenos Aires, 1836, Bonpland signature, Number 155 . B: Number 154, delivery note of two copies of the Geological Catalogue to Europe, note $1^{\circ}$. to the Natural History Museum in Paris and note $2^{\circ}$. to Humboldt, Buenos Aires December $26^{\text {th }}$, 1836 , signs Bonpland. Catalog continuation note Number 155.

Las tres muestras registradas por Bonpland de las Islas Malvinas e Isla de los Estados, Tierra del Fuego, Argentina, en su Catálogo Geológico 1858, tras paciente búsqueda digital en las colecciones minerológicas del Catálogo de Geología General del Museo Nacional de Historia Natural (MNHN), París, se pudo establecer que las mismas fueron estudiadas y clasificadas, como: 1) Objeto Fósil MNHN-GG-GG2013-29 Etiqueta: Turba de color marrón que conserva textura de la planta. Colección Bonpland, origen del país: Argentina Localidad: Islas Malvinas; 2) Objeto Roca MNHN-GG-GG2013-28 Etiqueta: Pirita cuprosa. Colección Bonpland, origen del país: Argentina Localidad: Islas de los Estados; y 3) Objeto Roca MNHN-GG-GG2013-21
Etiqueta: serpentina verdosa con diallage metaloide. Colección Bonpland, origen del país: Argentina Localidad: Islas de los Estados.

El enlace y la descripción original de cada uno de los registros geológicos en el MNHN, París, Francia, se encuentra disponible en: http://coldb.mnhn.fr/catalognumber/mnhn/gg/ gg2013-29; https://science.mnhn.fr/institution/ $\mathrm{mnhn} /$ collection/gg/item/gg2013-28; https:// science.mnhn.fr/institution/mnhn/collection/ gg/item/gg2013-21

En relación a las cartas de Bonpland a Humboldt, se señala que durante su permanencia en Buenos Aires, desde el mes de marzo a octubre de 1832, escribió cinco cartas, la primera de ellas, fechada el 7 de mayo de 1832, donde menciona por ejemplo, que 
recibió la hospitalidad del editor y periodista napolitano Pedro de Angelis (1784-1859), "Je me trouve ici dans la maison de M. le chevalier de Angelis, Napolitain, qui m'a reçu avec la plus grande hospitalité et que tu as vu jadis à Paris dans la société de madame la comtesse Orloff'; estoy aquí en la casa del caballero de Angelis, napolitano, quien me recibió con la mayor hospitalidad y a quien ha visto antes en París, en la sociedad de la señora condesa Orloff. La segunda carta es del 1 de junio, y expresa su deseo de visitar, entre otros destinos, la costa de Patagonia y las Islas Malvinas, "je suis souvent tenté de visiter toute la côte Patagonie, les isles Malouines, le Tucuman, le Chili et même, si cela m'était possible, d'aller au détroit de Magellan par terre"; a menudo me siento tentado a visitar toda la costa Patagónica, las Islas Malvinas, el Tucumán, Chile e incluso, si fuera posible, ir por tierra al Estrecho de Magallanes (Hossard, 2016).

La tercera carta a Humboldt, es del 12 de julio de 1832, la más extensa, que refiere a la situación planteada entre el Gobierno Argentino y el de los Estados Unidos, que tuvieron amplia repercusión en la opinión pública y una importante cobertura en los periódicos y semanarios locales, por los sucesos de la fragata USS Lexington, en Puerto Soledad, Malvinas, el 28 de diciembre de 1831 (Giudici, 2020). Aquí Bonpland tuvo una mirada crítica y pesimista sobre sus resultados, "le gouvernement actuel est tristement occupé des Isles Malouines avec les États-Unis du Nord et plongé dans ce mauvais pas, dont il sortira avec les [jambes cassées] malgré sa jactance dans les journaux; il se prépare une affaire plus désagréable encore avec le gouvernement français"; el gobierno actual está tristemente ocupado por las Islas Malvinas con los Estados Unidos y se lanzó a este mal paso, del cual saldrá con (las piernas rotas) no obstante su jactancia en los diarios; se prepara un asunto más desagradable todavía con el gobierno de Francia (Hamy, 1906).

Finalmente, la más breve de las cartas se corresponde con la del 14 de julio; y una última, en agosto de 1832 (Hossard, 2016), poco antes de salir de Buenos Aires con destino a San Borja (Brasil) por la costa del
Río Uruguay, donde informa a Humboldt que si bien aún piensa en un viaje por la costa Patagónica, ya no lo hace en relación a las Islas Malvinas, quizás como un presagio de la ocupación británica de 1833 .

\section{Conclusiones}

La puesta en valor del Catálogo Geológico del naturalista francés Aimé Bonpland, conservado desde 1858, como MS 210 en el MNHN de París, que contiene la descripción de rocas, minerales y fósiles, del sector de la América Meridional que exploró durante sus viajes, a partir de 1817, cuando llegó a Buenos Aires, Argentina; permitió, mediante la comprobación de fuentes documentales históricas, el hallazgo, identificación, descripción y clasificación de las muestras geológicas de las Islas Malvinas e Isla de los Estados, Tierra del Fuego, Argentina. Las muestras fueron incorporadas al mencionado catálogo por Bonpland, en 1832, cuando las recibió de Luis Vernet, primer gobernador y comandante argentino de las Islas Malvinas; circunstancias históricas que ponen en perspectiva el valioso patrimonio natural de la República Argentina.

\section{Agradecimientos}

Se agradece al Concejo Deliberante de la Municipalidad de Paso de los Libres, Corrientes, por el dictado de la Resolución $N^{\circ}$ 034/19, que Declaró de Interés Municipal el trabajo de investigación realizado por el Historiador David N. F. Guevara, sobre el sabio francés Aimé Bonpland. Del mismo modo por su apoyo: a la Fundación Yayetopa, Instituto de Investigación Bonpland, Paso de los Libres, Corrientes; a la Dra. María Silvia Ferrucci, Dra. Myriam Carolina Peichoto y Lic. María Cecilia Puigbó, IBONE, Corrientes. También, a los que colaboraron en las traducciones en lengua francesa: Traductor e Intérprete Robinson Savary desde París, Francia; Profesora Gabriela Sesma de la Alianza Francesa, Río Grande, Tierra del Fuego; a Diego Libedinsky, UNNE, Resistencia, Chaco y a quienes lo hicieron 
en lengua inglesa: Tatiana Rudiander Conte, Profesora en Lengua y Literatura Inglesa Facultad de Humanidades y Ciencias de la Educación Universidad Nacional de La Plata y a la Profesora de inglés Silvina Origgi, de Paso de los Libres, Corrientes. También se agradece al Dr. en Geología Rogelio Daniel Acevedo, CADIC, Ushuaia, Tierra del Fuego y al Dr. Alberto Gómez Gutiérrez, PhD FLS, Profesor Titular Instituto de Genética Humana Facultad de Medicina, Bogotá, Colombia. Al equipo del Museo de Farmacobotánica, UBA, Marcelo Luis Wagner, Leonardo M. Anconatani, Micaela Pereira y Melina Reabis. Al personal y a la curadora Sra. Véronique Van de Ponseele de la Biblioteca Central del MNHN de París; al investigador francés Dr. Cédric Cerruti y a la Sra. Delia del Carmen (Cota) Velázquez de Tierra del Fuego, Argentina. Finalmente, al Dr. Rodrigo Cajade, UNNE, Corrientes; a la Lic. Aurora Arbelo y al Sr. Juan Ignacio Bonpland, de la Fundación Amado Bonpland, Corrientes y a los descendientes de la familia Bonpland, Paso de los Libres, Corrientes.

\section{Bibliografía}

BONPLAND, A. (1858). MS 210 Catalogue pour servir a la Géologie des cótes de l'Uruguay, du Parana, de la plata; de toutes les missions jesuitiques, des provinces du Paraguay, de Corrientes, de l'EntreRios de la Cisplatine et de la république argentine, par Aimé Bonpland. Bibliothèque Centrale du Muséum National d'Histoire Naturelle, París, Francia.
BRUNEL, A. (1871). Biographie d'Aimé Bonpland. Troisième èdition. L. Guérin \& Cie, ed., París. https://doi.org/10.5962/bhl.title.57895

CERRUTI, C. (2013). Un sabio acorrentinado: Amado Bonpland, un hombre al servicio de la ciencia y de Corrientes.1er. Ed. Moglia Ediciones, Corrientes.

GIUDICI, T. M. (2020). Malvinas en la prensa argentina: de la creación de la Comandancia Política y Militar a los sucesos del USS Lexington. https://www.cancilleria. gob.ar/userfiles/ut/11-tomas_m_giudici.pdf

GUEVARA, D. N. F. (2016). Julio Popper el Alquimista de El Páramo - Tierra del Fuego 1885-1893. Instituto Geográfico Nacional, Argentina.

GUEVARA, D. N. F. (2019). Amado Bonpland, una actividad minera poco conocida del sabio francés, en busca de las minas de mercurio en La Cruz y Tres Cerros, Corrientes y su aporte a la geología y mineralogía en tiempos de la Confederación Argentina. En ARBELO DE MAZZARO, A., G. D. MANGO DE RUBIO \& J. A. PERSI (eds.), II Congreso Internacional Interdisciplinario Aimé Bonpland-Alexander von Humboldt Año 2017, pp. 123-142. Instituto de la Cultura, Moglia Ediciones, Corrientes.

HAMY, E. T. (1906). Aimé Bonpland, médecin et naturaliste, explorateur de l'Amérique du Sud; sa vie, son oeuvre, sa correspondance avec un choix de pièces relatives à sa biographie, un portrait et une carte. E. Guilmoto, París.

https://doi.org/10.5962/bhl.title.60911

HOSSARD, N. (2016). Alexander von Humboldt \& Aimé Bonpland. Correspondence 1805-1858. L'Harmattan, París.

LOURTEIG, A. (1977). Aimé Bonpland. Bonplandia 3: 269-317. https://doi.org/10.30972/bon.3162590 